\title{
General Dirichlet series, arithmetic convolution equations and Laplace transforms
}

\author{
by \\ Helge Glöckner (Paderborn), Lutz G. Lucht (Goslar) and \\ ŠTeFAn PorubskÝ (Praha)
}

\begin{abstract}
In the earlier paper [Proc. Amer. Math. Soc. 135 (2007)], we studied solutions $g: \mathbb{N} \rightarrow \mathbb{C}$ to convolution equations of the form

$$
a_{d} * g^{* d}+a_{d-1} * g^{*(d-1)}+\cdots+a_{1} * g+a_{0}=0,
$$

where $a_{0}, \ldots, a_{d}: \mathbb{N} \rightarrow \mathbb{C}$ are given arithmetic functions associated with Dirichlet series which converge on some right half plane, and also $g$ is required to be such a function. In this article, we extend our previous results to multidimensional general Dirichlet series of the form $\sum_{x \in X} f(x) e^{-s x}\left(s \in \mathbb{C}^{k}\right)$, where $X \subseteq[0, \infty)^{k}$ is an additive subsemigroup. If $X$ is discrete and a certain solvability criterion is satisfied, we determine solutions by an elementary recursive approach, adapting an idea of Fečkan [Proc. Amer. Math. Soc. 136 (2008)]. The solution of the general case leads us to a more comprehensive question: Let $X$ be an additive subsemigroup of a pointed, closed convex cone $C \subseteq \mathbb{R}^{k}$. Can we find a complex Radon measure on $X$ whose Laplace transform satisfies a given polynomial equation whose coefficients are Laplace transforms of such measures?
\end{abstract}

1. Results on general Dirichlet series and Laplace transforms. Let $k \in \mathbb{N}$ and $X \subseteq[0, \infty)^{k}$ be an infinite additive semigroup of $k$-tuples $x=\left(x_{1}, \ldots, x_{k}\right)$ with $0=(0, \ldots, 0) \in X$. Assume until further notice that $X$ is discrete (i.e., without a cluster point in $\mathbb{R}^{k}$ ) and hence countable. In this situation, the set $\mathcal{A}=\mathcal{A}(X):=\mathbb{C}^{X}$ of all arithmetic functions $g: X \rightarrow \mathbb{C}$ is a commutative complex algebra, called the Dirichlet algebra of $X$, under the usual linear operations and the convolution $*: \mathcal{A}^{2} \rightarrow \mathcal{A}$ as the algebra multiplication which for arbitrary functions $g, h \in \mathcal{A}$ is defined by

$$
(g * h)(x)=\sum_{\substack{x^{\prime}, x^{\prime \prime} \in X \\ x^{\prime}+x^{\prime \prime}=x}} g\left(x^{\prime}\right) h\left(x^{\prime \prime}\right) \quad(x \in X) .
$$

2000 Mathematics Subject Classification: 11A25, 44A10, 46H30.

Key words and phrases: arithmetic function, Dirichlet convolution, polynomial equation, analytic equation, topological algebra, holomorphic functional calculus, implicit function theorem, Laplace transform, semigroup, complex measure. 
The unit element $u \in \mathcal{A}$ under $*$ is given by $u(0)=1$ and $u(x)=0$ for non-zero $x \in X$, and the multiplicative group $\mathcal{A}^{*}$ of $\mathcal{A}$ with respect to the convolution consists of all functions $g \in \mathcal{A}$ satisfying $g(0) \neq 0$. For brevity, we write $g^{* j}$ for the convolution $g * \cdots * g$ with $j$ factors $g \in \mathcal{A}, g^{* 0}:=u$, and $g^{-1}$ for the inverse of $g$, i.e., $g * g^{-1}=u$.

Given $g \in \mathcal{A}$, consider the $k$-dimensional Dirichlet series

$$
\widetilde{g}(s)=\sum_{x \in X} g(x) e^{-x \cdot s} \quad\left(s \in \mathbb{C}^{k}\right)
$$

with the inner $\left({ }^{1}\right)$ product $x \cdot s=x_{1} s_{1}+\cdots+x_{k} s_{k}$ for $x=\left(x_{1}, \ldots, x_{k}\right) \in X$ and $s=\left(s_{1}, \ldots, s_{k}\right) \in \mathbb{C}^{k}$. For fixed $X$, these multidimensional series form an algebra which is isomorphic to $\mathcal{A}$ under ${ }^{\sim}: g \mapsto \widetilde{g}$ for $\widetilde{g}(s) \cdot \widetilde{h}(s):=(g * h)^{\sim}(s)$.

The aim of this paper is to investigate existence and analytic behavior of the solutions $g \in \mathcal{A}$ to the equation $T g=0$ for convolution polynomials $T: \mathcal{A} \rightarrow \mathcal{A}$ of degree $d \in \mathbb{N}$ defined by

$$
T g=a_{d} * g^{* d}+a_{d-1} * g^{*(d-1)}+\cdots+a_{1} * g+a_{0}
$$

with given arithmetic functions $a_{d}, a_{d-1}, \ldots, a_{1}, a_{0} \in \mathcal{A}, a_{d} \neq 0$.

Our first theorem concerns the existence of solutions $g \in \mathcal{A}$ to $T g=0$.

Theorem 1. For $T$ as in (1.2), let the polynomial $f(z) \in \mathbb{C}[z]$ be defined by

$$
f(z)=a_{d}(0) z^{d}+a_{d-1}(0) z^{d-1}+\cdots+a_{1}(0) z+a_{0}(0) .
$$

If $z_{0}$ is a simple zero of $f(z)$, then there exists a uniquely determined solution $g \in \mathcal{A}$ to $T g=0$ satisfying $g(0)=z_{0}$. If $f(z)$ has no simple zeros, then $\mathrm{Tg}=0$ need not possess any solution. In any case $\mathrm{Tg}=0$ has at most $d$ solutions $g \in \mathcal{A}$.

Section 2 contains an elementary proof extending that of [9] from $\mathcal{A}=$ $\mathcal{A}(\log \mathbb{N})$ to $\mathcal{A}(X)$ with $X \subseteq[0, \infty)^{k}$ a general discrete additive semigroup.

Given $x=\left(x_{1}, \ldots, x_{k}\right) \in \mathbb{R}^{k}$, define its size as $|x|:=\left|x_{1}\right|+\cdots+\left|x_{k}\right|$. For the study of absolute convergence of Dirichlet series $\widetilde{g}(s)$ it is convenient to consider the Banach algebras $\mathcal{A}_{r}$ of arithmetic functions $g \in \mathcal{A}$ with bounded $r$-norm

$$
\|g\|_{r}:=\sum_{x \in X}|g(x)| e^{-r|x|}
$$

for $r \in \mathbb{R}$, and also the algebra

$$
\mathcal{A}_{\infty}:=\bigcup\left\{\mathcal{A}_{r}: r \in \mathbb{R}\right\} .
$$

$\left({ }^{1}\right)$ Because $x$ is real, we omit complex conjugation. 
Let $H_{r}:=\{s \in \mathbb{C}: \operatorname{Re} s>r\}$ and $\bar{H}_{r}$ be its closure. Given $g \in \mathcal{A}_{r}$, the series $\widetilde{g}(s)$ converges absolutely for $s \in \bar{H}_{r}^{k}$. We shall use the same symbol, $\widetilde{g}(s)$, also for the value of the sum.

The next theorem guarantees that under suitable conditions solutions $g \in \mathcal{A}$ to $T g=0$ belong to $\mathcal{A}_{\infty}$, if $a_{d}, \ldots, a_{0} \in \mathcal{A}_{\infty}$.

TheOREM 2. Suppose that $a_{d}, a_{d-1}, \ldots, a_{1}, a_{0} \in \mathcal{A}_{\infty}$ in (1.2), with $a_{d} \neq 0$. If $z_{0}$ is a simple zero of $f(z)$ in (1.3), then there exists a solution $g \in \mathcal{A}_{\infty}$ to $T g=0$ satisfying $g(0)=z_{0}$.

In Section 3 we give an elementary proof of Theorem 2 extending that of Fečkan [5] in the case $\mathcal{A}=\mathcal{A}(\log \mathbb{N})$ to the multidimensional case $\mathcal{A}(X)$.

As in [9], Theorem 2 is the special case

$$
m=1, \quad F: \mathbb{C}^{d+1} \times \mathbb{C} \rightarrow \mathbb{C}, \quad F\left(w_{0}, \ldots, w_{d}, z\right)=\sum_{j=0}^{d} w_{j} z^{j}
$$

of a multidimensional version:

Theorem 3. For open subsets $V \subseteq \mathbb{C}^{n}$ and $Z \subseteq \mathbb{C}^{m}$, let

$$
F: V \times Z \rightarrow \mathbb{C}^{m}, \quad(v, z) \mapsto F(v, z),
$$

be a holomorphic function and $\left(v_{0}, z_{0}\right) \in V \times Z$ be such that $F\left(v_{0}, z_{0}\right)=0$. Let $a_{1}, \ldots, a_{n} \in \mathcal{A}=\mathcal{A}(X)$ satisfy the condition $\left(a_{1}(0), \ldots, a_{n}(0)\right)=v_{0}$. If the differential $\partial_{z} F\left(v_{0}, z\right)$ at $z=z_{0}$ is in $\mathrm{GL}_{m}(\mathbb{C})$, then there exists a unique $m$-tuple $\left(g_{1}, \ldots, g_{m}\right) \in \mathcal{A}^{m}$ such that

$$
\begin{aligned}
\left(g_{1}(0), \ldots, g_{m}(0)\right) & =z_{0}, \\
F\left[a_{1}, \ldots, a_{n}, g_{1}, \ldots, g_{m}\right] & =0 .
\end{aligned}
$$

If , in addition, $a_{1}, \ldots, a_{n} \in \mathcal{A}_{\infty}$, then also $g_{1}, \ldots, g_{m} \in \mathcal{A}_{\infty}$ and both

$$
\left(\widetilde{a}_{1}(s), \ldots, \widetilde{a}_{n}(s), \widetilde{g}_{1}(s), \ldots, \widetilde{g}_{m}(s)\right) \in V \times Z
$$

and

$$
F\left(\widetilde{a}_{1}(s), \ldots, \widetilde{a}_{n}(s), \widetilde{g}_{1}(s), \ldots, \widetilde{g}_{m}(s)\right)=0
$$

hold for all $s \in \bar{H}_{r}^{k}$, with $r$ sufficiently large. In this case, also (1.4), (1.6) and (1.7) uniquely determine $\left(g_{1}, \ldots, g_{m}\right) \in \mathcal{A}_{\infty}^{m}$.

Here the left hand side of (1.5) is obtained via multivariable holomorphic functional calculus (see [2] for a recent exposition in the required generality).

In Section 4 a proof of Theorem 3 is given based on a version of the implicit function theorem proved by Biller [2] and techniques taken from the theory of commutative topological algebras.

The results concerning generalized Dirichlet series are special cases of results concerning Laplace transforms (proved in Section 6), which we outline now. The necessary background material concerning convex cones and 
Laplace transforms (of positive measures) needed here can be found, e.g., in [10] or [8].

In the following, let $C \subseteq \mathbb{R}^{k}$ be a non-empty closed convex cone $\left({ }^{2}\right)$.

Assume that $C$ is pointed $\left({ }^{3}\right)$ and generating $\left({ }^{4}\right)$. Since $C$ is pointed, the dual cone $C^{\star}:=\left\{y \in \mathbb{R}^{k}: x y \geq 0\right.$ for all $\left.x \in C\right\}$ has non-empty interior (see [10, Proposition V.1.5(ii)]). We pick a $y_{0} \in\left(C^{\star}\right)^{\circ}$. Then $x y_{0}>0$ for each $x \in C \backslash\{0\}$ (cf. [10, Proposition V.1.4(v)]).

Let $X \subseteq C$ be an arbitrary (not necessarily discrete or countable) additive subsemigroup with $0 \in X$, equipped with a Hausdorff topology which makes the inclusion map $X \rightarrow \mathbb{R}^{k}$ continuous and turns $X$ into an additive topological semigroup (i.e., addition $X \times X \rightarrow X$ is continuous).

Consider the complex vector space $M(X)$ of all complex (not necessarily bounded) Radon measures $\mu$ on $X$, defined on the $\delta$-ring $\delta(X)$ generated by the set of compact subsets of $X$ (see Section 5 for our measure-theoretic setting). For $\mu \in M(X)$, let $|\mu| \in M_{+}(X)$ denote the associated total variation measure. Given $r \in \mathbb{R}$, let $M_{r}(X)$ be the set of all $\mu \in M(X)$ such that

$$
\|\mu\|_{r}:=\int_{X} e^{-r x y_{0}} d|\mu|(x)<\infty .
$$

To emphasize the dependence on $y_{0}$, we occasionally write $M_{r}^{\left(y_{0}\right)}(X)$ instead of $M_{r}(X)$ and $\|\mu\|_{r}^{\left(y_{0}\right)}$ instead of $\|\mu\|_{r}$. Then $M_{r}(X)$ is a vector subspace of $M(X)$ and the convolution of measures turns $M_{r}(X)$ into a complex algebra. In fact, $\left(M_{r}(X),\|\cdot\|_{r}\right)$ is a commutative Banach algebra with unit element $\delta_{0}$ (point mass at $0 \in X$ ). It is isomorphic to the Banach algebra $M_{0}(X)$ of bounded complex Radon measures on $X$ via $\mu \mapsto e^{-r x y_{0}} d \mu(x)$, where we write $f(x) d \mu(x)$ or $f \odot \mu$ for the measure of density $f$ with respect to $\mu$.

From $M_{r}(X) \subseteq M_{t}(X)$ for $r \leq t$ we conclude that $M_{\infty}(X):=\bigcup_{r \in \mathbb{R}} M_{r}(X)$ is an algebra under convolution and is independent of the choice of $y_{0}$. Given $\mu \in M_{r}(X)$, the set

$$
D_{\mu}:=\left\{s \in \mathbb{C}^{k}: \int_{X} e^{-x \operatorname{Re} s} d|\mu|(x)<\infty\right\}
$$

is convex (cf. [10, Proposition V.4.3]), where Re $s$ stands for the real part of $s$. We define the Fourier-Laplace transform $\widetilde{\mu}: D_{\mu} \rightarrow \mathbb{C}$ of $\mu$ by

$$
\widetilde{\mu}(s):=\int_{X} e^{-x \cdot s} d \mu(x) .
$$

\footnotetext{
$\left({ }^{2}\right)$ That is, $C$ is convex and $[0, \infty) \cdot C \subseteq C$.

$\left({ }^{3}\right)$ That is, $C \cap(-C)=\{0\}$ or, equivalently, $C$ contains no lines [10, Corollary V.1.11].

$\left(^{4}\right)$ That is, $C-C=\mathbb{R}^{k}$ or, equivalently, $C$ has non-empty interior $C^{\circ}$ (cf. $[10$, Proposition 5.1.4(ii)]).
} 
Then $r y_{0}+C^{\star}+i \mathbb{R}^{k} \subseteq D_{\mu}$, entailing that the convex set $D_{\mu} \subseteq \mathbb{C}^{k}$ has non-empty interior. Given $\mu \in M_{r}(X)$, we write

$$
\widetilde{\mu}(\infty):=\mu(\{0\}) \text {. }
$$

For $r \in \mathbb{R}$ and a closed unital subalgebra $A \subseteq M_{0}(X)$, we set $A_{r}:=$ $\left\{e^{r x y_{0}} d \mu(x): \mu \in A\right\}$ and define $A_{\infty}:=\bigcup_{r \in \mathbb{R}} A_{r}$. Typically, $A=M_{0}(X)$.

TheOrem 4. Let $V \subseteq \mathbb{C}^{n}$ and $Z \subseteq \mathbb{C}^{m}$ be open subsets, the function

$$
F: V \times Z \rightarrow \mathbb{C}^{m}, \quad(v, z) \mapsto F(v, z),
$$

be holomorphic, and $\left(v_{0}, z_{0}\right) \in V \times Z$ with $F\left(v_{0}, z_{0}\right)=0$. Let $\lambda_{1}, \ldots, \lambda_{n} \in$ $A_{\infty}$ be measures such that $\left(\widetilde{\lambda}_{1}(\infty), \ldots, \widetilde{\lambda}_{n}(\infty)\right)=v_{0}$. If the differential $\partial_{z} F\left(v_{0}, z\right)$ at $z=z_{0}$ is in $\mathrm{GL}_{m}(\mathbb{C})$, then there exists a unique $m$-tuple $\mu=\left(\mu_{1}, \ldots, \mu_{m}\right) \in A_{\infty}^{m}$ such that

$$
\left(\widetilde{\mu}_{1}(\infty), \ldots, \widetilde{\mu}_{m}(\infty)\right)=z_{0}
$$

and, for some $r \in \mathbb{R}$,

$$
\left(\widetilde{\lambda}_{1}(s), \ldots, \widetilde{\lambda}_{n}(s), \widetilde{\mu}_{1}(s), \ldots, \widetilde{\mu}_{m}(s)\right) \in V \times Z
$$

holds for all $s \in r y_{0}+C^{\star}+i \mathbb{R}^{k}$ as well as

$$
F\left(\widetilde{\lambda}_{1}(s), \ldots, \widetilde{\lambda}_{n}(s), \widetilde{\mu}_{1}(s), \ldots, \widetilde{\mu}_{m}(s)\right)=0 .
$$

Here $\mu$ is also determined by (1.8) and the condition that

$$
F\left[\lambda_{1}, \ldots, \lambda_{n}, \mu_{1}, \ldots, \mu_{m}\right]=0
$$

holds in the commutative Banach algebra $A_{r}$ for some $r \in \mathbb{R}$ such that $\lambda_{1}, \ldots, \lambda_{n}, \mu_{1}, \ldots, \mu_{m} \in A_{r}$.

The preceding theorem subsumes the following special cases.

EXAMPLE 1. As before, let $C \subseteq \mathbb{R}^{k}$ be a pointed and generating closed convex cone. Let $X \subseteq C$ be an arbitrary subsemigroup such that $0 \in X$, and endow $X$ with the discrete topology. For fixed $y_{0} \in\left(C^{\star}\right)^{\circ}$ and $r \in \mathbb{R}$, define the weight $w: X \rightarrow \mathbb{R}_{+}$by $w(x)=e^{-r x y_{0}}$ and the $r$-norm $\|f\|_{r}$ of $f: X \rightarrow \mathbb{C}$ by

$$
\|f\|_{r}:=\sum_{x \in X} w(x)|f(x)|,
$$

where the right hand side denotes the supremum in $[0, \infty]$ of the finite partial sums. Then $\ell_{w}^{1}(X):=\left\{f \in \mathbb{C}^{X}:\|f\|_{r}<\infty\right\}$ is the complex vector space of functions of finite norm, and Theorem 4 applies to $X$. Note that the map

$$
M(X) \rightarrow \mathbb{C}^{X}, \quad \mu \mapsto f_{\mu},
$$

with $f_{\mu}(x):=\mu(\{x\})$ is an isomorphism of vector spaces which induces an isomorphism $M_{r}(X) \cong \ell_{w}^{1}(X)$. Given $\mu$ and $f=f_{\mu}$ as before, we have

$$
\|\mu\|_{r}=\sum_{x \in X}|f(x)| e^{-r x y_{0}}=\|f\|_{r} .
$$


If $\mu \in M_{r}(X)$, then $\|\mu\|_{r}<\infty$ and hence $f(x)=0$ for all but countably many $x$ (cf. $[11,4.15])$. Then

$$
\widetilde{\mu}(s)=\widetilde{f}(s):=\sum_{x \in X} f(x) e^{-s x}
$$

for all $s \in D_{\mu}$, where the right hand side is interpreted as the limit of an absolutely summable family of vectors (cf. [4, Chapter V, §3]).

Example 2. If $C=[0, \infty)^{k}$ in Example 1, then $C^{\star}=[0, \infty)^{k}$ with $y_{0}:=(1,1, \ldots, 1)$ in its interior and $r y_{0}+C^{\star}+i \mathbb{R}^{k}=\bar{H}_{r}^{k}$ for each $r \in \mathbb{R}$. If we equip an arbitrary subsemigroup $X \subseteq[0, \infty)^{k}$ containing 0 with the discrete topology, and take $A:=M_{0}(X)$, Theorem 4 provides a generalization of the second half of Theorem 3 , which does not require discreteness of $X$ (nor countability).

EXAMPLE 3. Theorem 4 also applies to an arbitrary additive semigroup $X \subseteq C$ endowed with the topology induced by $\mathbb{R}^{k}$. For example, we can let $X:=C$ be a pointed and generating closed convex cone in $\mathbb{R}^{k}$, with the induced topology.

EXAMPLE 4. If the semigroup $X \subseteq C$ is a Borel measurable subset of $\mathbb{R}^{k}$ (e.g., if $X=C$ ), we can equip $X$ with the topology induced by $\mathbb{R}^{k}$ and choose $A:=\mathbb{C} \delta_{0}+L^{1}(X, \lambda) \odot \lambda$, where $\lambda$ denotes the restriction of Lebesgue-Borel measure on $\mathbb{R}^{k}$ to $\delta(X)$.

2. Proof of Theorem 1. For the proof of Theorem 1, we arrange the elements $x$ of $X$ according to their size $|x|$ and, in case of equal size, in lexicographic order of the components. In this way, we obtain a total order $\preceq$ on $X$. Since $X$ is discrete, the number of elements of $X$ having the same size is finite. Furthermore, it easily follows that $(X, \preceq)$ is order isomorphic to $(\mathbb{N}, \leq)$, enabling us to argue by induction on $x \in X$.

Rewrite $T g=0$ as an infinite system of equations

$$
\sum_{\substack{y, x^{\prime} \in X \\ y+x^{\prime}=x}}\left(a_{d}(y) g^{* d}\left(x^{\prime}\right)+a_{d-1}(y) g^{*(d-1)}\left(x^{\prime}\right)+\cdots+a_{0}(y) u\left(x^{\prime}\right)\right)=0
$$

for $x \in X$. It follows from (2.1) at $x=0$ that necessarily $f(g(0))=0$. Each simple zero $z_{0}$ of $f(z)$ serves for starting the following recurrence relation with $g(0)=z_{0}$.

Now let $0 \neq x \in X$. By separating all terms containing $g(x)$ in (2.1), we see that the coefficient of $g(x)$ equals the value of the derivative $f^{\prime}(z)$ at $z=z_{0}$. Therefore (2.1) takes the form 


$$
f^{\prime}(g(0)) \cdot g(x)=-\sum_{0 \leq j \leq d} \sum_{\begin{array}{c}
y, x_{1}, \ldots, x_{j} \in X \\
y+x_{1}+\cdots+x_{j}=x \\
x_{1}, \ldots, x_{j} \neq x
\end{array}} a_{j}(y) g\left(x_{1}\right) \cdots g\left(x_{j}\right)
$$

for $0 \neq x \in X$. Due to the choice of $g(0)=z_{0}$ we have $f^{\prime}(g(0)) \neq 0$. Note that $\left|x_{1}\right|, \ldots,\left|x_{j}\right|<|x|$ in all summands on the right hand side in (2.2) so that (2.2) represents a recursion formula, which uniquely determines an arithmetic function $g \in \mathcal{A}$.

To prove the second part of Theorem 1 , let $q \neq 0$ be an element of $X$ of minimal size $|q|>0$. Then there are only two additive decompositions of $q=x+x^{\prime}$ into two summands, namely $q+0$ and $0+q$. Let $a \in \mathcal{A}$ satisfy $a(0)=0 \neq a(q)$ and suppose that $T g=g * g-a$ has a zero $g \in \mathcal{A}$. Then $f(z)=z^{2}$ vanishes at the double zero $z=0$ only. It follows from $T g(0)=0$ that $g(0)=0$ and hence $T g(q)=2 g(0) g(q)-a(q)=-a(q) \neq 0$, a contradiction. Therefore $T g=0$ is unsolvable.

Since $\mathcal{A}$ is an integral domain, the polynomial $T g$ of degree $d$ has at most $d$ zeros $g \in \mathcal{A}$.

As an immediate consequence we note

COROLlary 1. If $f(z)$ satisfies $\operatorname{deg} f=d$ and all zeros of $f(z)$ are simple, then, with the distinct solutions $g_{1}, \ldots, g_{d} \in \mathcal{A}$ to $T g=0$, we have

$$
T g=a_{d} *\left(g-g_{1}\right) * \cdots *\left(g-g_{d}\right) .
$$

3. Elementary proof of Theorem 2. The Banach algebras $\mathcal{A}_{\varrho}$ and $\mathcal{A}:=\mathcal{A}_{0}$ are isomorphic under the map $a(x) \mapsto e^{-\varrho|x|} a(x)$ for $x \in X$, $a \in \mathcal{A}_{\varrho}$. For the proof of Theorem 2 we may therefore assume that $\left\|a_{j}\right\|:=$ $\left\|a_{j}\right\|_{0}<\infty$. It suffices to show that the solution $g \in \mathcal{A}$ to $T g=0$ with $f\left(z_{0}\right)=0$ and $f^{\prime}\left(z_{0}\right) \neq 0$ at $z_{0}=g(0)$ belongs to $\mathcal{A}_{r}$ for some $r \geq 0$. With $g_{r}(x):=e^{-r|x|} g(x)$ we rewrite $(2.2)$ as

$$
\begin{aligned}
& f^{\prime}\left(z_{0}\right) \cdot g_{r}(x)=-\sum_{0 \leq j \leq d} \sum_{\begin{array}{c}
y, x_{1}, \ldots, x_{j} \in X \\
y+x_{1}+\cdots+x_{j}=x \\
x_{1}, \ldots, x_{j} \neq x
\end{array}} \frac{a_{j}(y)}{e^{r|y|}} g_{r}\left(x_{1}\right) \cdots g_{r}\left(x_{j}\right) \\
& =-\sum_{0 \leq j \leq d} a_{j}(0) \sum_{\begin{array}{c}
x_{1}, \ldots, x_{j} \in X \\
x_{1}+\cdots, x_{j}=x \\
x_{1}, \ldots, x_{j} \neq x
\end{array}} g_{r}\left(x_{1}\right) \cdots g_{r}\left(x_{j}\right) \\
& -\sum_{0 \leq j \leq d} \sum_{\begin{array}{c}
y, x_{1}, \ldots, x_{j} \in X \\
y+x_{1}+\cdots+x_{j}=x \\
y \neq 0, x_{1}, \ldots, x_{j} \neq x
\end{array}} e^{-r|y|} a_{j}(y) g_{r}\left(x_{1}\right) \cdots g_{r}\left(x_{j}\right)
\end{aligned}
$$

for $0 \neq x \in X$. 
We have to prove that there is an $r \geq 0$ such that the partial sums

$$
S_{r}(m):=\sum_{0<|x| \leq m}\left|g_{r}(x)\right|
$$

are uniformly bounded for numbers $m$ belonging to the discrete image set $M=\left\{m_{0}, m_{1}, \ldots\right\}$ of $X$ under || with $m_{0}=0<m_{1}<\cdots$, say. Clearly $S_{r}\left(m_{0}\right)=0$, and for $n \in \mathbb{N}$ the above representation of $f^{\prime}\left(z_{0}\right) \cdot g_{r}(x)$ yields

$$
\begin{aligned}
\left|f^{\prime}\left(z_{0}\right)\right| \cdot S_{r}\left(m_{n}\right) & \leq \sum_{0 \leq j \leq d}\left|a_{j}(0)\right| \sum_{0<|x| \leq m_{n}} \sum_{\begin{array}{c}
x_{1}, \ldots, x_{j} \in X \\
x_{1}+\cdots+x_{j}=x \\
x_{1}, \ldots, x_{j} \neq x
\end{array}}\left|g_{r}\left(x_{1}\right)\right| \cdots\left|g_{r}\left(x_{j}\right)\right| \\
& +\sum_{0 \leq j \leq d} \sum_{\substack{0<|x| \leq m_{n} \\
m_{n}}} \sum_{\begin{array}{c}
y, x_{1}, \ldots, x_{j} \in X \\
y+x_{1}+\cdots+x_{j}=x \\
y \neq 0, x_{1}, \ldots, x_{j} \neq x
\end{array}} e^{-r|y|}\left|a_{j}(y)\right|\left|g_{r}\left(x_{1}\right)\right| \cdots\left|g_{r}\left(x_{j}\right)\right| .
\end{aligned}
$$

Let $\Sigma_{1}$ and $\Sigma_{2}$ denote the multiple sums on the right hand side of this inequality. By extracting all powers of $\left|g_{r}(0)\right|=|g(0)|=\left|z_{0}\right|$ from the inner $j$-fold sum of $\Sigma_{1}$, we first obtain

$$
\begin{aligned}
& \Sigma_{1} \leq \sum_{j=2}^{d}\left|a_{j}(0)\right| \sum_{i=2}^{j}\left(\begin{array}{l}
j \\
i
\end{array}\right)\left|z_{0}\right|^{j-i} \sum_{0<|x| \leq m_{n}} \sum_{\begin{array}{c}
x_{1}^{\prime}, \ldots, x_{i}^{\prime} \in X \\
x_{1}^{\prime}+\cdots, x_{i}^{\prime}=x \\
x_{1}^{\prime}, \ldots, x_{i}^{\prime} \neq 0, x
\end{array}}\left|g_{r}\left(x_{1}^{\prime}\right)\right| \cdots\left|g_{r}\left(x_{i}^{\prime}\right)\right| \\
& \leq \sum_{j=2}^{d}\left|a_{j}(0)\right| \sum_{i=2}^{j}\left(\begin{array}{l}
j \\
i
\end{array}\right)\left|z_{0}\right|^{j-i} S_{r}^{i}\left(m_{n-1}\right) .
\end{aligned}
$$

Next note that $e^{-r|y|} \leq e^{-r m_{1}}$ for all $y \in X, y \neq 0$. Then similarly

$$
\begin{aligned}
\Sigma_{2} & \leq e^{-r m_{1}} \sum_{0 \leq j \leq d} \sum_{0<|x| \leq m_{n}} \sum_{\substack{y, x_{1}, \ldots, x_{j} \in X \\
y+x_{1}+\cdots+x_{j}=x \\
y \neq 0, x_{1}, \ldots, x_{j} \neq x}}\left|a_{j}(y)\right|\left|g_{r}\left(x_{1}\right)\right| \cdots\left|g_{r}\left(x_{j}\right)\right| \\
& \leq e^{-r m_{1}}\left(\left\|a_{0}\right\|+\sum_{1 \leq j \leq d}\left\|a_{j}\right\| \sum_{\substack{x_{1}, \ldots, x_{j} \in X \\
\left|x_{1}+\cdots+x_{j}\right|<m_{n}}}\left|g_{r}\left(x_{1}\right)\right| \cdots\left|g_{r}\left(x_{j}\right)\right|\right) \\
& \leq e^{-r m_{1}} \sum_{j=0}^{d}\left\|a_{j}\right\|\left(\left|z_{0}\right|+S_{r}\left(m_{n-1}\right)\right)^{j} .
\end{aligned}
$$

Introduce polynomials $P(t), Q(t) \in \mathbb{R}[t]$ by

$$
P(t):=\frac{1}{\left|f^{\prime}\left(z_{0}\right)\right|} \sum_{j=2}^{d}\left|a_{j}(0)\right| \sum_{i=2}^{j}\left(\begin{array}{l}
j \\
i
\end{array}\right)\left|z_{0}\right|^{j-i} t^{i}, \quad Q(t):=\frac{1}{\left|f^{\prime}\left(z_{0}\right)\right|} \sum_{j=0}^{d}\left\|a_{j}\right\| t^{j}
$$


and summarize:

$$
S_{r}\left(m_{n}\right) \leq P\left(S_{r}\left(m_{n-1}\right)\right)+e^{-r m_{1}} Q\left(\left|z_{0}\right|+S_{r}\left(m_{n-1}\right)\right) \quad(n \in \mathbb{N}) .
$$

This is a recursive estimate starting with $S_{r}\left(m_{0}\right)=0$. It remains to show that there exist constants $r \geq 0$ and $C>0$ such that $S_{r}\left(m_{n}\right) \leq C$ for all $n \in \mathbb{N}$.

Since $P(t) \geq 0$ and $Q(t)>0$ are increasing functions of $t \in[0, \infty)$ with $\operatorname{deg} P(t) \leq \operatorname{deg} Q(t)=d$ or $P(t)=0$ (null function), it suffices by (3.1) to find solutions $r \geq 0$ and $t>0$ of the inequality

$$
P(t)+e^{-r m_{1}} Q\left(\left|z_{0}\right|+t\right) \leq t
$$

or, equivalently, of

$$
e^{-r m_{1}} \leq \frac{t-P(t)}{Q\left(\left|z_{0}\right|+t\right)}=: R(t) .
$$

Note that $P(0)=P^{\prime}(0)=0$ and $R$ is bounded above on $[0, \infty)$. Hence there exists some $t>0$ with $R(t)>0$. Choosing $r_{0}>0$ such that $e^{-r_{0} m_{1}} \leq R(t)$ we obtain (3.2). Hence $S_{r_{0}}\left(m_{n}\right) \leq t$ for all $n \in \mathbb{N}$, which completes the proof.

REMARK 1. The proof of Theorem 2 also leads to the quantitative estimates $\|g\|_{r} \leq C$ and $r=\varrho+m_{1}^{-1} \max \{0,-\log C\}$ with $C=\sup \{R(t): t \geq 0\}$.

For $X=\mathbb{N}_{0}^{k}$, Theorem 2 applies to multidimensional power series

$$
\widetilde{g}(w)=\sum_{n \in \mathbb{N}_{0}^{k}} g(n) w^{n}
$$

with coefficient sequences $g \in \mathcal{A}(X)$ and $w^{n}=e^{-n \cdot s}$ for $n \in X, s \in \mathbb{C}^{k}$. We recover a special case of the implicit function theorem for complex analytic maps:

COROLlary 2. Let the power series $\widetilde{a}_{0}(w), \ldots, \widetilde{a}_{d-1}(w)$ and $\widetilde{a}_{d}(w) \neq 0$ be holomorphic functions of $w \in \mathbb{C}^{k}$ in a neighborhood of the origin. Suppose that $z_{0} \in \mathbb{C}$ is a simple zero of the polynomial

$$
a_{d}(0) z^{d}+a_{d-1}(0) z^{d-1}+\cdots+a_{0}(0) \in \mathbb{C}[z] .
$$

Then there exists a local solution $\widetilde{g}(w)$ with $\widetilde{g}(0)=z_{0}$ to

$$
\widetilde{a}_{d}(w) \widetilde{g}^{d}(w)+\widetilde{a}_{d-1}(w) \widetilde{g}^{d-1}(w)+\cdots+\widetilde{a}_{0}(w)=0
$$

that is again holomorphic in a neighborhood of the origin.

For $X=(\log \mathbb{N})^{k}$, Theorem 2 also applies to multidimensional ordinary Dirichlet series

$$
\widetilde{g}(s)=\sum_{n \in \mathbb{N}^{k}} g(n) n^{-s}
$$


with coefficient sequences $g \in \mathcal{A}\left(\mathbb{N}^{k}\right)$ and $n^{-s}=e^{-\left(s_{1} \log n_{1}+\cdots+s_{k} \log n_{k}\right)}$ for $n \in \mathbb{N}^{k}$ and $s \in \mathbb{C}^{k}$ (cf. [9, Theorem 3]). With $1:=(1, \ldots, 1) \in \mathbb{N}^{k}$ we obtain

Corollary 3. Let the $k$-dimensional Dirichlet series $\widetilde{a}_{0}(s), \ldots, \widetilde{a}_{d-1}(s)$ and $\widetilde{a}_{d}(s) \neq 0$ converge absolutely for all $s$ in some $H_{r}^{k}$. Suppose that $z_{0} \in \mathbb{C}$ is a simple zero of the polynomial

$$
a_{d}(1) z^{d}+a_{d-1}(1) z^{d-1}+\cdots+a_{0}(1) \in \mathbb{C}[z] .
$$

Then there exists a Dirichlet series $\widetilde{g}(s)$ with $g(1)=z_{0}$ that solves

$$
\widetilde{a}_{d}(s) \widetilde{g}^{d}(s)+\widetilde{a}_{d-1}(s) \widetilde{g}^{d-1}(s)+\cdots+\widetilde{a}_{0}(s)=0
$$

and also converges absolutely for all $s$ in some $H_{\varrho}^{k}$.

4. Proof of Theorem 3. Our proof of Theorem 3 involves general facts concerning analytic equations in topological algebras. Recall that a complex topological algebra is an algebra $A$ over $\mathbb{C}$, equipped with a locally convex vector topology making the bilinear algebra multiplication $A \times A \rightarrow A$ a continuous map. It is called complete if the underlying locally convex space is complete. A continuous inverse algebra is a unital, associative complex topological algebra $A$ whose group of units $A^{*}$ is open in $A$ and whose inversion map $A^{*} \rightarrow A, a \mapsto a^{-1}$, is continuous (see [2], [7] and [12]). The spectrum of a commutative continuous inverse algebra $A$ is the set $\widehat{A}$ of all unital algebra homomorphisms $\xi: A \rightarrow \mathbb{C}$. It is known that $\xi \mapsto \operatorname{ker} \xi$ is a bijection from $\widehat{A}$ onto the set of all maximal (proper) ideals of $A$ (cf. [2, Lemma 2.5]). The spectrum of an element $a \in A$ is defined as $\sigma(a):=$ $\left\{s \in \mathbb{C}: s-a \notin A^{*}\right\}$, and by [2, Theorem 2.7(a)], it coincides with the set $\{\xi(a): \xi \in \widehat{A}\}$. The joint spectrum of elements $a_{1}, \ldots, a_{n} \in A$ is defined as

$$
\sigma\left(a_{1}, \ldots, a_{n}\right):=\left\{\left(\xi\left(a_{1}\right), \ldots, \xi\left(a_{n}\right)\right): \xi \in \widehat{A}\right\} \subseteq \mathbb{C}^{n} .
$$

Then $\sigma\left(a_{1}, \ldots, a_{n}\right) \subseteq \sigma\left(a_{1}\right) \times \cdots \times \sigma\left(a_{n}\right)$. If $A$ is a commutative, complete continuous inverse algebra, $a_{1}, \ldots, a_{n} \in A$ and $f: U \rightarrow \mathbb{C}$ a holomorphic function on an open subset $U \subseteq \mathbb{C}^{n}$ such that $\sigma\left(a_{1}, \ldots, a_{n}\right) \subseteq U$, then the holomorphic functional calculus gives rise to an element $f\left[a_{1}, \ldots, a_{n}\right] \in A$ (see $[2, \S 4]$ for details).

REMARK 2. The following simple facts are essential for our purposes:

(a) (Naturality of the holomorphic functional calculus) If $A, f$ and $a_{1}, \ldots, a_{n} \in A$ are as before and $\phi: A \rightarrow B$ is a continuous homomorphism of unital algebras to a complete, commutative continuous inverse algebra $B$, then $\sigma\left(\phi\left(a_{1}\right), \ldots, \phi\left(a_{n}\right)\right) \subseteq \sigma\left(a_{1}, \ldots, a_{n}\right)$ and

$$
\phi\left(f\left[a_{1}, \ldots, a_{n}\right]\right)=f\left[\phi\left(a_{1}\right), \ldots, \phi\left(a_{n}\right)\right]
$$

(see $[2$, Theorem 4.9]). 
(b) If $A=\mathbb{C}$, then $f\left[a_{1}, \ldots, a_{n}\right]=f\left(a_{1}, \ldots, a_{n}\right)$ is the value of $f$ at $\left(a_{1}, \ldots, a_{n}\right) \in U \subseteq \mathbb{C}^{n}$.

Our proof of Theorem 3 uses the following special case of Biller [2, Theorem 8.2], applied to algebras whose spectrum is a singleton.

Lemma 1. Let $A$ be a complete, commutative continuous inverse algebra whose spectrum is a singleton, $\widehat{A}=\{\xi\}$. Let $V \subseteq \mathbb{C}^{n}$ and $Z \subseteq \mathbb{C}^{m}$ be open sets and let $F: V \times Z \rightarrow \mathbb{C}^{m}$ be a holomorphic function. Suppose that $v_{0} \in V$ and $z_{0} \in Z$ are such that $F\left(v_{0}, z_{0}\right)=0$ and $\left.\partial_{z} F\left(v_{0}, z\right)\right|_{z=z_{0}} \in \mathrm{GL}_{m}(\mathbb{C})$. Then, for each $\left(a_{1}, \ldots, a_{n}\right) \in A^{n}$ satisfying $\left(\xi\left(a_{1}\right), \ldots, \xi\left(a_{n}\right)\right)=v_{0}$, there exists a unique $\left(g_{1}, \ldots, g_{m}\right) \in A^{m}$ such that $\left(\xi\left(g_{1}\right), \ldots, \xi\left(g_{m}\right)\right)=z_{0}$ and

$$
F\left[a_{1}, \ldots, a_{n}, g_{1}, \ldots, g_{m}\right]=0 .
$$

Given an infinite discrete additive semigroup $X \subseteq[0, \infty)^{k}$, the Dirichlet algebra $\mathcal{A}=\mathbb{C}^{X}$ satisfies the hypotheses of Lemma 1 when equipped with the product topology.

Lemma 2. $\mathcal{A}$ is a commutative continuous inverse algebra whose spectrum is a singleton, namely $\widehat{\mathcal{A}}=\{\xi\}$ with $\xi: \mathcal{A} \rightarrow \mathbb{C}, f \mapsto f(0)$. Furthermore, $\mathcal{A}$ is a Fréchet space (and hence complete).

Proof. First note that $\mathcal{A}^{*}=\{f \in \mathcal{A}: f(0) \neq 0\}$, which is an open subset of $\mathcal{A}$. To see this, let $f \in \mathcal{A}$. The function $\xi$ described in the lemma is a unital algebra homomorphism to $\mathbb{C}$. Hence, if $f \in \mathcal{A}$ and $f(0)=\xi(f)=0$, then $f$ is not invertible. If, on the other hand, $f(0) \neq 0$, then the equation $f * g=u$ has a unique solution $g$ in $\mathcal{A}$, by Theorem 1 (and then $g=f^{-1}$ ). Given $x \in X \backslash\{0\}$, the proof of Theorem 1 shows that $f^{-1}(x)$ only depends on $f(y)$ for $y$ in the finite set $\{y \in X:|y| \leq|x|\}$. Moreover, $f^{-1}(x)$ is a rational (and hence continuous) function in the $f(y)$. Therefore the inversion map $\mathcal{A}^{*} \rightarrow \mathcal{A}, f \mapsto f^{-1}$, is continuous and thus $\mathcal{A}$ is a continuous inverse algebra. Since $\mathcal{A} \backslash \operatorname{ker} \xi=\mathcal{A}^{*}$, it follows that every proper ideal of $\mathcal{A}$ is contained in $\operatorname{ker} \xi$. Hence, if $\eta \in \widehat{\mathcal{A}}$, then $\operatorname{ker} \eta=\operatorname{ker} \xi$ (since $\operatorname{ker} \eta$ is a maximal ideal) and thus $\eta=\xi$. Hence $\widehat{\mathcal{A}}=\{\xi\}$. Being a countably infinite power of the Fréchet space $\mathbb{C}$, also $\mathcal{A}=\mathbb{C}^{X}$ is a Fréchet space.

In connection with Remark 2(a), the following lemma will be useful.

Lemma 3. If $X$ is discrete, then the inclusion map $\lambda: \mathcal{A}_{r} \rightarrow \mathcal{A}$ is a continuous algebra homomorphism, for each $r \in \mathbb{R}$.

Proof. Since $\mathcal{A}_{r}$ is a subalgebra of $\mathcal{A}$, the inclusion map is an algebra homomorphism. Because $\lambda$ is linear and $|\lambda(f)(x)|=|f(x)| \leq e^{r y_{0} x}\|f\|_{r}$, we see that $\mathcal{A}_{r} \rightarrow \mathbb{C}, f \mapsto \lambda(f)(x)$, is continuous for each $x \in X$. Since $\mathcal{A}=\mathbb{C}^{X}$ is equipped with the product topology, this implies that $\lambda$ is continuous. 


\section{Proof of Theorem 3.}

SteP 1. By Lemma $2, \mathcal{A}$ is a complete, commutative continuous inverse algebra with spectrum $\{\xi\}$. Since $\left(\xi\left(a_{1}\right), \ldots, \xi\left(a_{n}\right)\right)=\left(a_{1}(0), \ldots, a_{n}(0)\right)=$ $v_{0}$, Lemma 1 shows the existence and uniqueness of $\left(g_{1}, \ldots, g_{m}\right) \in \mathcal{A}^{m}$ such that conditions (1.4) and (1.5) of Theorem 3 are satisfied.

To complete the proof, we shall use Theorem 4 established below (the proof of which is independent of Theorem 3 ).

SteP 2. If $a_{1}, \ldots, a_{n} \in \mathcal{A}_{\infty}$, then Theorem 4 (combined with Example 1) shows that there is a uniquely determined $m$-tuple $\left(g_{1}, \ldots, g_{m}\right) \in \mathcal{A}_{\infty}^{m}$ such that (1.4) and (1.5) hold in some $\mathcal{A}_{r}$. The elements $\left(g_{1}, \ldots, g_{m}\right) \in \mathcal{A}_{\infty}^{m}$ coincide with the corresponding elements of $\mathcal{A}$ obtained in Step 1. To see this, pick $r \in \mathbb{R}$ with $a_{1}, \ldots, a_{n}, g_{1}, \ldots, g_{m} \in \mathcal{A}_{r}$ and $F\left[a_{1}, \ldots, a_{n}, g_{1}, \ldots, g_{m}\right]=0$ in $\mathcal{A}_{r}$. Since the inclusion map $\lambda: \mathcal{A}_{r} \rightarrow \mathcal{A}$ is a continuous algebra homomorphism (by Lemma 3 ), we obtain

$$
0=\lambda\left(F\left[a_{1}, \ldots, a_{n}, g_{1}, \ldots, g_{m}\right]\right)=F\left[\lambda\left(a_{1}\right), \ldots, \lambda\left(a_{n}\right), \lambda\left(g_{1}\right), \ldots, \lambda\left(g_{m}\right)\right]
$$

due to the naturality of holomorphic functional calculus (see Remark 2(a)). Now the uniqueness assertion from Step 1 applies.

STEP 3. In view of (1.12), the validity of (1.6) and (1.7) for large $r$ follows from (1.9) and (1.10) in Theorem 4.

SteP 4. In Theorem 4, (1.8), (1.9) and (1.10) imply (1.11). Hence, as a special case, (1.4), (1.6) and (1.7) imply (1.5) and thus determine $\left(g_{1}, \ldots, g_{m}\right)$.

5. Technical preliminaries. The measures required for our purposes are (possibly unbounded!) complex Radon measures. Since a suitable reference describing the relevant aspects of their theory does not seem to be available, we add this section for the convenience of readers with a standard knowledge of measure theory. Various results on Laplace transforms are also provided. Our main sources are [1], [3], and [6].

The measure-theoretic setting. Given a Hausdorff topological space $X$, let $\delta(X)$ be the $\delta$-ring generated by the set $\mathcal{K}(X)$ of compact subsets of $X$ (thus $\delta(X)$ is the smallest set containing $\mathcal{K}(X)$ and closed under finite unions, relative complements and countable intersections). A function $\mu: \delta(X) \rightarrow \mathbb{C}$ is called a complex measure if $\mu(B)=\sum_{n=1}^{\infty} \mu\left(B_{n}\right)$ for all sequences $\left(B_{n}\right)_{n \in \mathbb{N}}$ of disjoint sets $B_{n} \in \delta(X)$ such that $B:=\bigcup_{n \in \mathbb{N}} B_{n} \in \delta(X)$. If, furthermore, $\mu(B) \in[0, \infty)$ for each $B \in \delta(X)$, then $\mu$ is called a positive measure. To any complex measure $\mu,[6$, Proposition II.1.3] associates a positive measure $|\mu|$ (the total variation measure). A complex measure $\mu$ is called a Radon measure if $|\mu|$ is inner regular, i.e., $|\mu|(B)=\sup _{K \in \mathcal{K}(B)}|\mu|(K)$. If $\mu$ is a positive Radon measure on $X$, then $\left.\mu\right|_{\mathcal{K}(X)}$ is a Radon content in the 
sense of [1, Definition 2.1.2] and hence extends uniquely to an inner regular measure $\bar{\mu}: \mathcal{B}(X) \rightarrow[0, \infty]$ on the Borel $\sigma$-algebra $\mathcal{B}(X)$ of $X$ (see [1, Theorem 2.1.4]). By abuse of notation, we shall frequently write $\mu$ in place of $\bar{\mu}$.

REMARK 3. Note that every measure $\mu$ gives rise to a family $\left(\mu_{K}\right)_{K \in \mathcal{K}(X)}$ of measures $\mu_{K}:=\left.\mu\right|_{\mathcal{B}(K)}$ which are compatible in the sense that $\left.\mu_{L}\right|_{\mathcal{B}(K)}=$ $\mu_{K}$ for all $K, L \in \mathcal{K}(X)$ with $K \subseteq L$. Here $\mu$ is positive if and only if each $\mu_{K}$ is positive. Since

$$
\delta(X)=\bigcup_{K \in \mathcal{K}(X)} \mathcal{B}(K),
$$

it is easy to see that, conversely, every compatible family $\left(\mu_{K}\right)_{K \in \mathcal{K}(X)}$ defines a complex Radon measure $\mu$ via $\left.\mu\right|_{\mathcal{B}(K)}:=\mu_{K}$ for $K \in \mathcal{K}(X)$.

If $\mu$ is a complex Radon measure on $X$, then $\mu_{K}$ is a bounded Radon measure for each $K \in \mathcal{K}(X)$ and thus $\mu_{K}=i \mu_{K}^{1}-\mu_{K}^{2}-i \mu_{K}^{3}+\mu_{K}^{4}$ with positive Radon measures $\mu_{K}^{j}, j \in\{1, \ldots, 4\}$, where $\mu_{K}^{4}$ and $\mu_{K}^{2}$ are the positive and negative variations of the real part of $\mu_{K}$, respectively, and $\mu_{K}^{1}$, $\mu_{K}^{3}$ are those of its imaginary part (see $[11, \S 6.6]$ ). Since $\mu_{K}^{j} \leq|\mu|_{K}$, the measure $\mu_{K}^{j}$ has a density with respect to the Radon measure $|\mu|_{K}$ (by the Radon-Nikodym theorem), entailing that $\mu_{K}^{j}$ is inner regular. By Remark 3, the families $\left(\mu_{K}^{j}\right)_{K \in \mathcal{K}(X)}$ determine positive Radon measures $\mu_{j}$ on $X$ for $j \in\{1, \ldots, 4\}$ such that $\mu_{j} \leq|\mu|$ and

$$
\mu=i \mu_{1}-\mu_{2}-i \mu_{3}+\mu_{4}
$$

We say that a Borel measurable function $f: X \rightarrow \mathbb{C}$ is $\mu$-integrable if $f \in$ $L^{1}(X,|\mu|)$. In this case, we write $f=\sum_{j=1}^{4} i^{j} f_{j}$ with $0 \leq f_{j} \in L^{1}(X,|\mu|)$ and $\mu=\sum_{k=1}^{4} i^{k} \mu_{k}$ with positive Radon measures $\mu_{k}$ such that $\mu_{k} \leq|\mu|$ and define $\left({ }^{5}\right)$

$$
\int_{X} f d \mu:=\sum_{j, k=1}^{4} i^{j+k} \int_{X} f_{j} d \mu_{k} .
$$

Let $M(X)$ be the space of complex Radon measures on $X$, let $M_{0}(X):=$ $\{\mu \in M(X):\|\mu\|:=|\mu|(X)<\infty\}$ be the space of bounded complex Radon measures, and $M_{+}(X)$ be the set of positive Radon measures. Then $\left(M_{0}(X),\|\cdot\|\right)$ is a Banach space, because the space of all bounded complex measures on $X$ is a Banach space (see [6, II.1.5]) and also $M(K)$ is a Banach space for each $K \in \mathcal{K}(X)$ (entailing, in view of Remark 3, that limits of complex Radon measures are again Radon).

$\left({ }^{5}\right)$ Typically, $f_{4}$ and $f_{2}$ (resp., $f_{1}$ and $f_{3}$ ) are the positive and negative parts of the real part (resp., imaginary part) of $f$, and $\mu_{1}, \ldots, \mu_{4}$ are as in (5.2). 
If $\mu \in M_{0}(X)$ in (5.2), then $\mu_{j} \in M_{0,+}(X):=M_{0}(X) \cap M_{+}(X)$ for each $j \in\{1, \ldots, 4\}$, and $\bar{\mu}:=i \bar{\mu}_{1}-\bar{\mu}_{2}-i \bar{\mu}_{3}+\bar{\mu}_{4}$ is the unique extension of $\mu$ to an (ordinary) complex measure on $(X, \mathcal{B}(X))$ whose total variation is inner regular. Again, we usually write $\mu$ instead of $\bar{\mu}$.

Given Hausdorff spaces $X_{j}$ for $j \in\{1,2\}$ and $\mu_{j} \in M_{+}(X)$, there exists a unique positive Radon measure $\mu_{1} \otimes \mu_{2}$ on $X_{1} \times X_{2}$ such that

$$
\left(\mu_{1} \otimes \mu_{2}\right)\left(B_{1} \times B_{2}\right)=\mu_{1}\left(B_{1}\right) \mu_{2}\left(B_{2}\right) \quad \text { for } B_{1} \in \mathcal{B}\left(X_{1}\right), B_{2} \in \mathcal{B}\left(X_{2}\right)
$$

(see [1, Corollary 2.1.11]). Since $\left(\mu_{1}+t \nu_{1}\right) \otimes \mu_{2}=\mu_{1} \otimes \mu_{2}+t\left(\nu_{1} \otimes \mu_{2}\right)$ and $\mu_{1} \otimes\left(\mu_{2}+t \nu_{2}\right)=\mu_{1} \otimes \mu_{2}+t\left(\mu_{1} \otimes \nu_{2}\right)$ for all $\mu_{j}, \nu_{j} \in M_{0,+}\left(X_{j}\right)$ and $t \geq 0$, where $M_{0}\left(X_{j}\right)$ is spanned by $M_{0,+}\left(X_{j}\right)$ as a complex vector space, standard arguments provide a unique complex bilinear map

$$
\beta: M_{0}\left(X_{1}\right) \times M_{0}\left(X_{2}\right) \rightarrow M_{0}\left(X_{1} \times X_{2}\right)
$$

such that $\beta\left(\mu_{1}, \mu_{2}\right)=\mu_{1} \otimes \mu_{2}$ for all $\mu_{j} \in M_{0,+}\left(X_{j}\right)$. We write $\mu_{1} \otimes \mu_{2}:=$ $\beta\left(\mu_{1}, \mu_{2}\right)$ also for general $\mu_{j} \in M_{0}\left(X_{j}\right)$. Then

$$
\left|\mu_{1} \otimes \mu_{2}\right| \leq\left|\mu_{1}\right| \otimes\left|\mu_{2}\right| \quad \text { for all } \mu_{1} \in M_{0}\left(X_{1}\right), \mu_{2} \in M_{0}\left(X_{2}\right) .
$$

To see this, note first that

$$
\left(\rho_{1} \odot \mu_{1}\right) \otimes\left(\rho_{2} \odot \mu_{2}\right)=\left(\rho_{1} \otimes \rho_{2}\right) \odot\left(\mu_{1} \otimes \mu_{2}\right)
$$

for all $\mu_{j} \in M_{+}\left(X_{j}\right)$ and Borel measurable functions $\rho_{j}: X_{j} \rightarrow[0, \infty]$ such that $\int_{K} \rho_{j} d \mu_{j}<\infty$ for each $K \in \mathcal{K}\left(X_{j}\right)$ for $j \in\{1,2\}$, where

$$
\rho_{1} \otimes \rho_{2}: X_{1} \times X_{2} \rightarrow \mathbb{C}, \quad\left(x_{1}, x_{2}\right) \mapsto \rho_{1}\left(x_{1}\right) \rho_{2}\left(x_{2}\right) .
$$

In fact, the right hand side of (5.5) is a positive Radon measure which satisfies the characterization of the product measure on the left (cf. (5.3)).

As a consequence of the Radon-Nikodym theorem, $\mu_{j}$ as in (5.4) admits a polar decomposition $\mu_{j}=\rho_{j} \odot\left|\mu_{j}\right|$ for a suitable measurable function $\rho_{j}: X_{j} \rightarrow \mathbb{C}$ such that $\left|\rho_{j}(x)\right|=1$ for each $x \in X_{j}$ (see [11, Theorem 6.12]). We write $\rho_{j}=\sum_{k=1}^{4} i^{k} \rho_{j}^{k}$ with $\rho_{j}^{1}:=\operatorname{Im}\left(\rho_{j}\right)_{+}, \rho_{j}^{2}:=\operatorname{Re}\left(\rho_{j}\right)_{-}, \rho_{j}^{3}:=\operatorname{Im}\left(\rho_{j}\right)_{-}$ and $\rho_{j}^{4}:=\operatorname{Re}\left(\rho_{j}\right)_{+}$. Then, by (5.5) and the bilinearity of $\otimes$ and $\odot$,

$$
\begin{aligned}
\mu_{1} \otimes \mu_{2} & =\sum_{k, l=1}^{4} i^{k+l}\left(\rho_{1}^{k} \odot\left|\mu_{1}\right|\right) \otimes\left(\rho_{2}^{l} \odot\left|\mu_{2}\right|\right) \\
& =\sum_{k, l=1}^{4} i^{k+l}\left(\rho_{1}^{k} \otimes \rho_{2}^{l}\right) \odot\left(\left|\mu_{1}\right| \otimes\left|\mu_{2}\right|\right) \\
& =\left(\rho_{1} \otimes \rho_{2}\right) \odot\left(\left|\mu_{1}\right| \otimes\left|\mu_{2}\right|\right),
\end{aligned}
$$

entailing that $\left|\mu_{1} \otimes \mu_{2}\right|=\left|\mu_{1}\right| \otimes\left|\mu_{2}\right|$. Thus (5.4) holds.

We now return to the situation described in Section 1 , where $C \subseteq \mathbb{R}^{k}$ is a pointed and generating closed convex cone and $X \subseteq C$ a (continuously 
embedded) topological semigroup with continuous addition

$$
\alpha: X \times X \rightarrow X, \quad\left(x_{1}, x_{2}\right) \mapsto x_{1}+x_{2} .
$$

It is useful to observe that each $\mu \in M_{r,+}(X):=M_{r}(X) \cap M_{+}(X)$ (with $M_{r}(X), y_{0}$ and $\|\cdot\|_{r}$ as in the introduction) is a $\sigma$-finite measure, since

$$
\int_{X} e^{-r x y_{0}} d \mu(x)=\|\mu\|_{r}<\infty,
$$

where $e^{-r x y_{0}}>0$ for each $x \in X$. Given $\mu_{1}, \mu_{2} \in M_{+}(X)$, we define their convolution as the image measure

$$
\mu_{1} * \mu_{2}:=\alpha\left(\mu_{1} \otimes \mu_{2}\right)
$$

on $\mathcal{B}(X)$; thus $\left(\mu_{1} * \mu_{2}\right)(B):=\left(\mu_{1} \otimes \mu_{2}\right)\left(\alpha^{-1}(B)\right)$. If $\mu_{1} * \mu_{2}$ is finite on compact sets, then $\mu_{1} * \mu_{2}$ is a positive Radon measure [1, Proposition 2.1.15]. In particular, $\mu_{1} * \mu_{2}$ is a positive Radon measure if $\mu_{1}, \mu_{2} \in M_{r,+}(X)$ for some $r \in \mathbb{R}$. In fact, for each compact set $K \subseteq X$, we have $a:=\inf \left\{e^{-r x y_{0}}: x \in K\right\}$ $>0$ and

$$
\begin{aligned}
a \mu(K) & \leq \int_{X} e^{-r x y_{0}} d\left(\mu_{1} * \mu_{2}\right)(x)=\int_{X} e^{-r x y_{0}} d \alpha\left(\mu_{1} \otimes \mu_{2}\right)(x) \\
& =\int_{X \times X} e^{-r \alpha\left(x_{1}, x_{2}\right) y_{0}} d\left(\mu_{1} \otimes \mu_{2}\right)\left(x_{1}, x_{2}\right) \\
& =\int_{X \times X} e^{-r x_{1} y_{0}} e^{-r x_{2} y_{0}} d\left(\mu_{1} \otimes \mu_{2}\right)\left(x_{1}, x_{2}\right) \\
& =\int_{X} e^{-r x_{1} y_{0}} d \mu_{1}\left(x_{1}\right) \int_{X} e^{-r x_{2} y_{0}} d \mu_{2}\left(x_{2}\right)<\infty
\end{aligned}
$$

(by transformation of integrals and Fubini's theorem) $\left({ }^{6}\right)$, whence $\mu(K)<\infty$. The previous calculation also shows that

$$
\mu_{1} * \mu_{2} \in M_{r}(X) \text { for all } \mu_{1}, \mu_{2} \in M_{r,+}(X) .
$$

Since $\left(\mu_{1}+t \nu_{1}\right) * \mu_{2}=\mu_{1} * \mu_{2}+t\left(\nu_{1} * \mu_{2}\right)$ and $\mu_{1} *\left(\mu_{2}+t \nu_{2}\right)=\mu_{1} * \mu_{2}+t\left(\mu_{1} * \nu_{2}\right)$ for all $\mu_{j}, \nu_{j} \in M_{r,+}(X)$ and $t \geq 0$, where $M_{r}(X)$ is spanned by $M_{r,+}(X)$ as a complex vector space, standard arguments provide a unique complex bilinear map

$$
M_{r}(X) \times M_{r}(X) \rightarrow M_{r}(X), \quad\left(\mu_{1}, \mu_{2}\right) \mapsto \mu_{1} * \mu_{2},
$$

extending the convolution of measures in $M_{r,+}(X)$ already defined.

If $\mu_{1}, \mu_{2} \in M_{0}(X)$, then

$$
\mu_{1} * \mu_{2}=\alpha\left(\mu_{1} \otimes \mu_{2}\right) .
$$

$\left({ }^{6}\right)$ Since $\mu_{1}, \mu_{2}$ are $\sigma$-finite measures and the map $X \times X \rightarrow \mathbb{C},\left(x_{1}, x_{2}\right) \mapsto$ $e^{-r x_{1} y_{0}} e^{-r x_{2} y_{0}}$, is $\mathcal{B}(X) \otimes \mathcal{B}(X)$-measurable, the standard Fubini theorem (as in [11, Theorem 8.12]) suffices; we do not need specialized versions for Radon measures like [1, Theorem 2.1.12]. 
In fact, if we redefine convolution via (5.10), then $\left|\mu_{1} * \mu_{2}\right| \leq\left|\mu_{1}\right| *\left|\mu_{2}\right|$ (as a consequence of (5.4)) and thus $\mu_{1} * \mu_{2} \in M_{0}(X)$ with

$$
\left\|\mu_{1} * \mu_{2}\right\| \leq\left(\left|\mu_{1}\right| *\left|\mu_{2}\right|\right)(X)=\left\|\mu_{1}\right\| \cdot\left\|\mu_{2}\right\| .
$$

Since $\mu_{1} * \mu_{2}$ from (5.10) coincides with the old definition in the case of positive measures and is bilinear in $\left(\mu_{1}, \mu_{2}\right)$, it coincides with the convolution defined in (5.9). It is clear from (5.10) that convolution is associative and commutative (because so is $\alpha$ ). Since also (5.11) holds and $\left\|\delta_{0}\right\|=1$, we see that $\left(M_{0}(X), *,\|\cdot\|\right)$ is a unital commutative Banach algebra. For each $r \in \mathbb{R}$, the map

$$
\phi: M_{r}(X) \rightarrow M_{0}(X), \quad \mu \mapsto e^{-r x y_{0}} d \mu(x),
$$

is a surjective linear isometry and an isomorphism of algebras. Hence also $\left(M_{r}(X), *,\|\cdot\|_{r}\right)$ is a unital commutative Banach algebra.

To complete our discussion of measures, let us show that $M_{\infty}(X)$ is indeed independent of the choice of $y_{0}$ (as claimed in the introduction).

LEMmA 4. If $y_{0}, y_{1} \in\left(C^{\star}\right)^{\circ}$, then $\bigcup_{r \in \mathbb{R}} M_{r}^{\left(y_{0}\right)}(X)=\bigcup_{r \in \mathbb{R}} M_{r}^{\left(y_{1}\right)}(X)$.

Proof. Since $y_{1} \in\left(C^{\star}\right)^{\circ}$, there exists $\varepsilon>0$ such that $c:=y_{1}-\varepsilon y_{0} \in C^{\star}$ and thus $y_{1}=\varepsilon y_{0}+c$. Then $r x y_{1}=r \varepsilon x y_{0}+r x c \geq r \varepsilon x y_{0}$ for each $r \geq 0$ and $x \in X$, whence $\|\mu\|_{r}^{\left(y_{1}\right)} \leq\|\mu\|_{r \varepsilon}^{\left(y_{0}\right)}$ for each $\mu \in M_{r \varepsilon}^{\left(y_{0}\right)}(X)$ and thus $M_{r \varepsilon}^{\left(y_{0}\right)}(X) \subseteq M_{r}^{\left(y_{1}\right)}(X)$. Hence $\bigcup_{r \in \mathbb{R}} M_{r}^{\left(y_{0}\right)}(X) \subseteq \bigcup_{r \in \mathbb{R}} M_{r}^{\left(y_{1}\right)}(X)$. The opposite inclusion can be shown analogously.

Some basic facts concerning Laplace transforms. Fourier-Laplace transforms have the following properties (part of which will be essential later).

Lemma 5. Let $C \subseteq \mathbb{R}^{k}$ be a pointed and generating closed convex cone, $X \subseteq C$ be a continuously embedded topological semigroup with $0 \in X$, and $y_{0} \in\left(C^{\star}\right)^{\circ}$. Let $r \in \mathbb{R}$ and $\mu \in M_{r}(X)$. Then the following holds:

(a) The function $\widetilde{\mu}$ is holomorphic on the interior of $D_{\mu}$.

(b) On ry $y_{0}+C^{\star}+i \mathbb{R}^{k}$, the function $\widetilde{\mu}$ is continuous.

(c) For each $\varepsilon>0$, there is $\rho \in[r, \infty)$ such that

$$
|\widetilde{\mu}(s)-\widetilde{\mu}(\infty)| \leq \varepsilon \quad \text { for each } s \in \rho y_{0}+C^{\star}+i \mathbb{R}^{k} .
$$

(d) If also $\nu \in M_{r}(X)$ and $\left.\widetilde{\mu}\right|_{U}=\left.\widetilde{\nu}\right|_{U}$ for some non-empty open set $U \subseteq \mathbb{C}^{k}\left(\right.$ or $\left.U \subseteq \mathbb{R}^{k}\right)$ such that $U \subseteq D_{\mu} \cap D_{\nu}$, then $\mu=\nu$.

Proof. (a) Write $\mu=i \mu_{1}-\mu_{2}-i \mu_{3}+\mu_{4}$ as a linear combination of positive measures, as in (5.2). Then $\widetilde{\mu}=i \widetilde{\mu}_{1}-\widetilde{\mu}_{2}-i \widetilde{\mu}_{3}+\widetilde{\mu}_{4}$ on $D_{\mu}^{0}$, where $D_{\mu}^{0} \subseteq D_{\mu_{j}}^{0}$ and $\widetilde{\mu}_{j}$ is holomorphic on $D_{\mu_{j}}^{0}$ for each $j \in\{1,2,3,4\}$, by [10, Proposition V.4.6]. 
(b) Let $\left(s_{n}\right)_{n \in \mathbb{N}}$ be a convergent sequence in $r y_{0}+C^{\star}+i \mathbb{R}^{k}$, with limit $s$. Then $\widetilde{\mu}\left(s_{n}\right) \rightarrow \widetilde{\mu}(s)$ as $n \rightarrow \infty$ by Lebesgue's dominated convergence theorem, using the integrable majorant $X \rightarrow[0, \infty), x \mapsto e^{-r x y_{0}}$.

(c) If $s_{n}=\rho_{n}+t_{n}$ with $t_{n} \in C^{\star}+i \mathbb{R}^{k}$ and $\rho_{n} \in[r, \infty)$ such that $\rho_{n} \rightarrow \infty$, then $e^{-s_{n} x} \rightarrow \mathbf{1}_{\{0\}}(x)$ (with $\mathbf{1}_{\{0\}}: X \rightarrow\{0,1\}$ the characteristic function of $\{0\})$ because $\left|e^{-s_{n} x}\right| \leq e^{-\rho_{n} x y_{0}}$, where $x y_{0}>0$ for $x \in X \backslash\{0\}$. Hence $\widetilde{\mu}\left(s_{n}\right) \rightarrow \int_{X} \mathbf{1}_{\{0\}}(x) d \mu(x)=\mu(\{0\})=\widetilde{\mu}(\infty)$, by dominated convergence with majorant $e^{-r x y_{0}}$.

(d) Suppose first that $\mu$ and $\nu$ are positive measures. Let $\lambda: X \rightarrow \mathbb{R}^{k}$ be the inclusion map and $\mu_{1}:=\lambda(\mu), \nu_{1}:=\lambda(\nu)$ be the image measures on $\mathbb{R}^{k}$ (equipped with the Borel $\sigma$-algebra). Then $\mu_{1}=\nu_{1}$ (for example, by [8, Theorem 14.11(e)]) and $\left.\mu\right|_{\mathcal{K}(X)}=\left.\mu_{1}\right|_{\mathcal{K}(X)}=\left.\nu_{1}\right|_{\mathcal{K}(X)}=\left.\nu\right|_{\mathcal{K}(X)}$. Consequently, $\mu=\nu$.

The general case amounts to injectivity of the linear map $M_{r}(X) \rightarrow \mathbb{C}^{U}$, $\left.\mu \mapsto \widetilde{\mu}\right|_{U}$, which we prove using an idea from [1, proof of Proposition 6.5.2]. Suppose that $\mu \in M_{r}(X)$ and $\left.\widetilde{\mu}\right|_{U}=0$. We write

$$
\mu=i \mu_{1}-\mu_{2}-i \mu_{3}+\mu_{4}
$$

with positive measures $\mu_{1}, \ldots, \mu_{4} \in M_{r}(X)$, as in (5.2). Then $\left.\widetilde{\mu}\right|_{D_{\mu}^{\circ}}=0$ by the identity theorem for analytic functions, and thus

$$
i \widetilde{\mu}_{1}(s)-\widetilde{\mu}_{2}(s)-i \widetilde{\mu}_{3}(s)+\widetilde{\mu}_{4}(s)=0 \quad \text { for all } s \in D_{\mu}^{\circ} .
$$

Since $D_{\mu}^{\circ}$ is invariant under the complex conjugation ${ }^{-}$, we see that also $0=\overline{\widetilde{\mu}(s)}=\widetilde{\bar{\mu}}(\bar{s})$ for all $s \in D_{\mu}^{\circ}$ and hence

$$
-i \widetilde{\mu}_{1}(s)-\widetilde{\mu}_{2}(s)+i \widetilde{\mu}_{3}(s)+\widetilde{\mu}_{4}(s)=0 \quad \text { for all } s \in D_{\mu}^{\circ} .
$$

Adding (5.12) and (5.13), we deduce that $\widetilde{\mu}_{2}(s)=\widetilde{\mu}_{4}(s)$ and hence also $\widetilde{\mu}_{1}(s)=\widetilde{\mu}_{3}(s)$, for all $s \in D_{\mu}^{\circ}$. Then $\mu_{1}=\mu_{3}$ and $\mu_{2}=\mu_{4}$ by the case of positive measures already discussed, and hence $\mu=0$.

The next lemma enables us to use the naturality of holomorphic functional calculus (see Remark 2(a)) in connection with Laplace transforms.

Lemma 6. For each $s \in r y_{0}+C^{\star}+i \mathbb{R}^{k}$, the map

$$
h_{s}: M_{r}(X) \rightarrow \mathbb{C}, \quad h_{s}(\mu):=\int_{X} e^{-s x} d \mu(x),
$$

is a continuous algebra homomorphism. Furthermore,

$$
h_{s}(\mu)=\widetilde{\mu}(s) .
$$

Proof. It is clear that $h_{s}$ is linear. Since $\left|h_{s}(\mu)\right| \leq \int_{X} e^{-r y_{0} x} d|\mu|(x)=$ $\|\mu\|_{r}$, the linear map $h_{s}$ is continuous. Since $h_{s}$ is linear and $M_{r}(X)$ is spanned by positive measures, $h_{s}\left(\mu_{1} * \mu_{2}\right)=h_{s}\left(\mu_{1}\right) h_{s}\left(\mu_{2}\right)$ will hold for arbitrary $\mu_{1}, \mu_{2} \in M_{r}(X)$ if we can prove it for positive measures $\mu_{1}, \mu_{2}$. In 
the latter case, repeating the calculation leading to (5.7), we obtain

$$
\begin{aligned}
h_{s}\left(\mu_{1} * \mu_{2}\right) & =\int_{X} e^{-s x} d\left(\mu_{1} * \mu_{2}\right)(x) \\
& =\int_{X} e^{-s x_{1}} d \mu_{1}\left(x_{1}\right) \int_{X} e^{-s x_{2}} d \mu_{2}\left(x_{2}\right)=h_{s}\left(\mu_{1}\right) h_{s}\left(\mu_{2}\right) .
\end{aligned}
$$

Since also $h_{s}\left(\delta_{0}\right)=1$, the map $h_{s}$ is a homomorphism of unital algebras.

The following consequence will be applied repeatedly.

Lemma 7. Let $\mu_{1}, \ldots, \mu_{n} \in M_{r}(X)$ and $f: \mathbb{C}^{n} \supseteq U \rightarrow \mathbb{C}$ be a holomorphic function such that $\nu:=f\left[\mu_{1}, \ldots, \mu_{n}\right]$ is defined in $M_{r}(X)$. Then

$$
\widetilde{\nu}(s)=f\left(\widetilde{\mu}_{1}(s), \ldots, \widetilde{\mu}_{n}(s)\right) \quad \text { for all } s \in r y_{0}+C^{\star}+i \mathbb{R}^{k} .
$$

Proof. Using Lemma 6 and the facts compiled in Remark 2, we obtain

$$
\begin{aligned}
\widetilde{\nu}(s) & =h_{s}(\nu)=h_{s}\left(f\left[\mu_{1}, \ldots, \mu_{n}\right]\right)=f\left[h_{s}\left(\mu_{1}\right), \ldots, h_{s}\left(\mu_{n}\right)\right] \\
& =f\left(\widetilde{\mu}_{1}(s), \ldots, \widetilde{\mu}_{n}(s)\right) .
\end{aligned}
$$

6. Proof of Theorem 4. The proof of Theorem 4 relies on the following lemma (where $A_{r}$ is as in the theorem). Given $\mu \in A_{r}$ and $t \geq r$, we write $\sigma_{t}(\mu)$ for the spectrum of $\mu$ in the commutative Banach algebra $A_{t}$ and, likewise, $\sigma_{t}\left(\mu_{1}, \ldots, \mu_{n}\right)$ for joint spectra in $A_{t}$. Given $\varepsilon>0$ and $z \in \mathbb{C}$, let $B_{\varepsilon}(z):=\{w \in \mathbb{C}:|w-z|<\varepsilon\}$.

Lemma 8. If $\mu \in A_{r}$, then

$$
\left\|\mu-\widetilde{\mu}(\infty) \delta_{0}\right\|_{t} \rightarrow 0 \quad \text { as } t \rightarrow \infty .
$$

For every $\varepsilon>0$, there exists $t_{0} \geq r$ such that $\sigma_{t}(\mu) \subseteq B_{\varepsilon}(\widetilde{\mu}(\infty))$ for all $t \geq t_{0}$.

Proof. If $\left\|\mu-\widetilde{\mu}(\infty) \delta_{0}\right\|_{t}<\varepsilon$, then $\sigma_{t}\left(\mu-\widetilde{\mu}(\infty) \delta_{0}\right) \subseteq B_{\varepsilon}(0)$ (see [11, Corollary 3 to Theorem 18.4]) and thus $\sigma_{t}(\mu) \subseteq B_{\varepsilon}(\widetilde{\mu}(\infty))$. Hence the second assertion follows if we can prove the first. To this end, let $\left(t_{n}\right)_{n \in \mathbb{N}}$ be a sequence of real numbers $t_{n} \geq r$ such that $t_{n} \rightarrow \infty$ as $n \rightarrow \infty$. Since $\left|\mu-\widetilde{\mu}(\infty) \delta_{0}\right|=|\mu|-|\mu|(\{0\}) \delta_{0}$, Lebesgue's dominated convergence theorem shows that

$$
\begin{aligned}
\left\|\mu-\widetilde{\mu}(\infty) \delta_{0}\right\|_{t_{n}} & =\int_{X} e^{-t_{n} x y_{0}} d|\mu|(x)-\int_{X} e^{-t_{n} x y_{0}}|\mu|(\{0\}) d \delta_{0}(x) \\
& =\int_{X \backslash\{0\}} e^{-t_{n} x y_{0}} d|\mu|(x) \rightarrow 0 \quad \text { as } n \rightarrow \infty ;
\end{aligned}
$$

we have used the facts that $e^{-t_{n} x y_{0}} \rightarrow 0$ as $n \rightarrow \infty$ for each $x \in X \backslash\{0\}$ and $e^{-t_{n} x y_{0}} \leq e^{-r x y_{0}}$, where $\int_{X \backslash\{0\}} e^{-r x y_{0}} d|\mu|(x) \leq\|\mu\|_{r}<\infty$. 
Proof of Theorem 4. Existence of $\mu$. By the implicit function theorem, there exist open neighborhoods $V_{0} \subseteq V$ of $v_{0}$ and $Z_{0} \subseteq Z$ of $z_{0}$ such that

$$
\left\{(v, z) \in V_{0} \times Z_{0}: F(v, z)=0\right\}=\operatorname{graph}(\phi)
$$

is the graph of a holomorphic function

$$
\phi=\left(\phi_{1}, \ldots, \phi_{m}\right): V_{0} \rightarrow Z_{0}
$$

with $\phi\left(v_{0}\right)=z_{0}$. After shrinking $V_{0}$, we may assume that

$$
\phi_{1}\left(V_{0}\right) \times \cdots \times \phi_{m}\left(V_{0}\right) \subseteq Z_{0} .
$$

Let $r \in \mathbb{R}$ be such that $\lambda_{1}, \ldots, \lambda_{n} \in A_{r}$. Since $V_{0}$ is an open neighborhood of $\left(\widetilde{\lambda}_{1}(\infty), \ldots, \widetilde{\lambda}_{n}(\infty)\right)$, Lemma 8 implies that, after increasing $r$ if necessary, we have

$$
\sigma_{t}\left(\lambda_{1}\right) \times \cdots \times \sigma_{t}\left(\lambda_{n}\right) \subseteq V_{0} \quad \text { for all } t \geq r
$$

Hence

$$
\mu_{j}:=\phi_{j}\left[\lambda_{1}, \ldots, \lambda_{n}\right] \in A_{r}
$$

can be defined using functional calculus in $A_{r}$, for $j \in\{1, \ldots, m\}$. Set $\mu:=$ $\left(\mu_{1}, \ldots, \mu_{m}\right)$. By the spectral mapping theorem [2, Corollary 4.10],

$$
\sigma_{r}\left(\mu_{j}\right)=\phi_{j}\left(\sigma_{r}\left(\lambda_{1}, \ldots, \lambda_{n}\right)\right) \subseteq \phi_{j}\left(V_{0}\right) .
$$

Then

$$
\begin{aligned}
\sigma_{r}\left(\lambda_{1}, \ldots, \lambda_{n}, \mu_{1}, \ldots, \mu_{m}\right) & \subseteq \sigma_{r}\left(\lambda_{1}\right) \times \cdots \times \sigma_{r}\left(\lambda_{n}\right) \times \sigma_{r}\left(\mu_{1}\right) \times \cdots \times \sigma_{r}\left(\mu_{m}\right) \\
& \subseteq V_{0} \times Z_{0} .
\end{aligned}
$$

Moreover,

$$
F\left[\lambda_{1}, \ldots, \lambda_{n}, \mu_{1}, \ldots, \mu_{m}\right]=\left(F \circ\left(\operatorname{id}_{V_{0}}, \phi\right)\right)\left[\lambda_{1}, \ldots, \lambda_{n}\right]=0
$$

by the spectral mapping theorem, since $F \circ\left(\mathrm{id}_{V_{0}}, \phi\right)=0$ by $(6.1)$.

Using Lemma 7, we deduce from (6.2) that

$$
\begin{aligned}
0 & =F\left[\lambda_{1}, \ldots, \lambda_{n}, \mu_{1}, \ldots, \mu_{m}\right]^{\sim}(s) \\
& =F\left(\widetilde{\lambda}_{1}(s), \ldots, \widetilde{\lambda}_{n}(s), \widetilde{\mu}_{1}(s), \ldots, \widetilde{\mu}_{m}(s)\right),
\end{aligned}
$$

for each $s \in r y_{0}+C^{\star}+i \mathbb{R}^{k}$. Thus (1.9) and (1.10) from Theorem 4 hold. In view of (6.1), the preceding equality also implies (1.8).

Uniqueness of $\mu$. Let $\nu=\left(\nu_{1}, \ldots, \nu_{m}\right) \in\left(A_{t}\right)^{m}$ for some $t \in \mathbb{R}$ such that (1.8), (1.9) and (1.10) hold, with $\mu$ replaced by $\nu$ and $r$ replaced by $t$. After increasing $r$ or $t$, we may assume that $r=t$. After increasing $r$ further if necessary, we can achieve (using Lemma 8) that

$$
\sigma_{r}\left(\nu_{1}\right) \times \cdots \times \sigma_{r}\left(\nu_{m}\right) \subseteq Z_{0} .
$$

Hence

$$
\zeta:=F\left[\lambda_{1}, \ldots, \lambda_{n}, \nu_{1}, \ldots, \nu_{m}\right]
$$


can be defined in $A_{r}$. For each $s \in r y_{0}+C^{\star}+i \mathbb{R}^{k}$, we have

$$
\widetilde{\zeta}(s)=F\left(\widetilde{\lambda}_{1}(s), \ldots, \widetilde{\lambda}_{n}(s), \widetilde{\nu}_{1}(s), \ldots, \widetilde{\nu}_{m}(s)\right)=0,
$$

by hypothesis and Lemma 7 . In view of (6.1), (6.3) and (6.4), we have

$$
\left(\widetilde{\nu}_{1}(s), \ldots, \widetilde{\nu}_{m}(s)\right)=\phi(s)=\left(\widetilde{\mu}_{1}(s), \ldots, \widetilde{\mu}_{m}(s)\right)
$$

for each $s \in r y_{0}+C^{\star}+i \mathbb{R}^{k}$. Hence $\nu=\mu$, by Lemma $5(\mathrm{~d})$.

Proof of the final assertion. Assume that $\left(\widetilde{\nu}_{1}(\infty), \ldots, \widetilde{\nu}_{m}(\infty)\right)=z_{0}$ and

$$
F\left[\lambda_{1}, \ldots, \lambda_{n}, \nu_{1}, \ldots, \nu_{m}\right]=0
$$

in some $A_{t}$. After increasing $r$ or $t$, we may again assume that $t=r$. Applying $h_{s}$ to (6.5) for $s \in r y_{0}+C^{\star}+i \mathbb{R}^{k}$, we see that (1.9) and (1.10) hold. Hence $\nu=\mu$, by what has just been shown.

Acknowledgements. The second author is indebted to the Grant Agency of the Czech Republic, Grant \# 201/07/0191, for support.

The third author was supported by the Grant Agency of the Czech Republic, Grant \# 201/07/0191, and by the Institutional Research Plan AV0Z10300504. For some of the period during which this work was carried out he also thanks the Clausthal University of Technology for support.

\section{References}

[1] Ch. Berg, J. P. R. Christensen and P. Ressel, Harmonic Analysis on Semigroups, Springer, New York, 1984.

[2] H. Biller, Analyticity and naturality of the multi-variable functional calculus, Exposition. Math. 25 (2007), 131-163.

[3] N. Bourbaki, Intégration. Chapitre IX, Hermann, Paris, 1969.

[4] J. Dieudonné, Foundations of Modern Analysis, Academic Press, New York and London, 1960.

[5] M. Fečkan, Note on arithmetic convolution equations, Proc. Amer. Math. Soc. 136 (2008), 453-459.

[6] J. M. G. Fell and R. S. Doran, Representations of *-Algebras, Locally Compact Groups, and Banach *-Algebraic Bundles, Volume 1, Academic Press, Boston, 1988.

[7] H. Glöckner, Algebras whose groups of units are Lie groups, Studia Math. 153 (2002), 147-177.

[8] —, Positive definite functions on infinite-dimensional convex cones, Mem. Amer. Math. Soc. 166 (2003), no. 789.

[9] H. Glöckner, L. G. Lucht and Š. Porubský, Solutions to arithmetic convolution equations, Proc. Amer. Math. Soc. 135 (2007), 1619-1629.

[10] K.-H. Neeb, Holomorphy and Convexity in Lie Theory, de Gruyter, Berlin, 2000.

[11] W. Rudin, Real and Complex Analysis, McGraw-Hill, New York, 1987. 
[12] L. Waelbroeck, Les algèbres à inverse continu, C. R. Acad. Sci. Paris 238 (1954), 640-641.

Institut für Mathematik

Universität Paderborn

Warburger Str. 100

33098 Paderborn, Germany

E-mail: glockner@math.upb.de

Institute of Computer Science

Academy of Sciences of the Czech Republic

Pod Vodárenskou věží 2

18207 Praha 8, Czech Republic

E-mail: Stefan.Porubsky@cs.cas.cz
Siemensstr. 1 38640 Goslar, Germany E-mail: lg.lucht@cintech.de

Received May 3, 2008 